Review Article

\title{
Novel Biomarkers of Abdominal Aortic Aneurysm Disease: Identifying Gaps and Dispelling Misperceptions
}

\author{
Demetrios Moris, ${ }^{1}$ Eleftherios Mantonakis, ${ }^{1}$ Efthymios Avgerinos, ${ }^{2}$ Marinos Makris, \\ Chris Bakoyiannis, ${ }^{1}$ Emmanuel Pikoulis, ${ }^{1}$ and Sotirios Georgopoulos ${ }^{1}$ \\ ${ }^{1}$ First Department of Surgery, Vascular Unit, "Laiko" General Hospital, National and Kapodistrian University of Athens, \\ Agiou Thoma 17, 11527 Athens, Greece \\ ${ }^{2}$ Division of Vascular Surgery, University of Pittsburgh, Pittsburgh, PA 15213-2582, USA \\ ${ }^{3}$ Imperial College, London SW7 2AZ, UK
}

Correspondence should be addressed to Eleftherios Mantonakis; lefman@gmail.com

Received 6 March 2014; Revised 29 April 2014; Accepted 4 May 2014; Published 20 May 2014

Academic Editor: Thomas Schmitz-Rixen

Copyright (C) 2014 Demetrios Moris et al. This is an open access article distributed under the Creative Commons Attribution License, which permits unrestricted use, distribution, and reproduction in any medium, provided the original work is properly cited.

\begin{abstract}
Abdominal aortic aneurysm (AAA) is a prevalent and potentially life-threatening disease. Early detection by screening programs and subsequent surveillance has been shown to be effective at reducing the risk of mortality due to aneurysm rupture. The aim of this review is to summarize the developments in the literature concerning the latest biomarkers (from 2008 to date) and their potential screening and therapeutic values. Our search included human studies in English and found numerous novel biomarkers under research, which were categorized in 6 groups. Most of these studies are either experimental or hampered by their low numbers of patients. We concluded that currently no specific laboratory markers allow screeing for the disease and monitoring its progression or the results of treatment. Further studies and studies in larger patient groups are required in order to validate biomarkers as cost-effective tools in the AAA disease.
\end{abstract}

\section{Introduction}

Abdominal aortic aneurysm (AAA) is a relatively common and potentially life-threatening disease, with a prevalence ranging from $4.1 \%$ to $11.5 \%$ in European populations [1]. The most feared complication of AAA disease is rupture, which often leads to the patient's death. While small aneurysms may rupture too, the risk is greater for bigger aneurysms, so early diagnosis through screening and prompt evaluation of which aneurysms are likely to rupture are key points in the management and research around AAA. No effective conservative treatment exists and, when decided to intervene, surgical correction remains the only effective and "finite" treatment of AAA, with the optimal timing for surgery being the main debatable point. Existing evidence indicates better overall outcome when repair of AAA is performed on elective basis compared to the emergency repair [2-4]. Thus screening programs of AAA have been initiated in developed countries such as UK [5]. Current guidelines recommend elective repair for aneurysms of more than $5.5 \mathrm{~cm}$ in diameter, while increased risk groups (women, smokers, and vascular hypertension and chronic airway patients) may benefit by a lower threshold of $5 \mathrm{~cm}[6]$.

Advances in the endovascular aneurysm repair (EVAR) technique over the last two decades have led to a major paradigm shift in surgical approach for aortic aneurysms [7, 8]. By providing lower perioperative mortality and morbidity, endovascular techniques are increasingly being preferred in the elective setting $[8,9]$. However, late complications of EVAR, such as endoleaks, may result in progression of the underlying aneurysm, even delayed rupture, thus mandating a lifelong surveillance program [10].

Traditional AAA screening, evaluation, and surveillance programs employ the use of imaging techniques such as CT angiogram (CTA), ultrasound sonography (US), or magnetic resonance imaging (MRI). Despite the proven efficacy of 
these imaging techniques, the cost associated with such programs can incur significant financial burdens to the health care systems [11-14], so alternative methods are continuously being

researched.

Biomarkers have attracted much attention in the field of aneurysm research and are defined as measurable molecules (peptides, proteins, and metabolic products) that express a specific biological process in the organism at a given time [15]. In order for a biomarker to be useful, it should be able either to detect the disease itself or to express its progression. So a useful biomarker should detect the presence of a subclinical aneurysm or be a measure of its size and expansion rate, thus predicting the risk of rupture. It is known that this risk increases when the observed expansion rate is greater than the expected [16]. Furthermore, a biomarker could define the optimal surveillance intervals and possibly identify pathogenic pathways which could guide monitoring and treatment [17]. We should not fail to mention that, in order for a biomarker to be employed in modern healthcare system, its use should be cost-effective. Some of the patients selected through this process should be pointed towards more focused screening by specialized imaging techniques. Circulating biomarkers thus present as attractive alternatives for screening and monitoring purposes particularly in healthcare systems which lack the infrastructure to support other primary or secondary screening programs. In this review, we aim to provide a summary of the emerging biomarkers implicated in AAA pathophysiology.

\section{Materials and Methods}

The MEDLINE/PubMed database was searched for publications with the medical subject heading "biomarkers in abdominal aortic aneurysms (AAA)." Two independent reviewers (D.M. and E.M.) performed the literature search, the study selection, and the data extraction. All the references from the identified articles were searched for relevant information. The starting date was January 2008, since, previously, bibliography was covered by the review of Urbonavicius et al. [17] and the end date of the review was April 2014. We focused on human studies that reported the presence of aneurysm, size, expansion rates, and risk of rupture and on new biomarkers and their potential value relevant to the management of AAA. We excluded articles that were not written in English or articles of which full-texts and abstracts were not accessible or comprehensive.

\section{Results}

Numerous studies were found and categorized in the following categories: biomarkers related to the extracellular matrix homeostasis or proteolysis, biomarkers related to cellular signaling pathways, proteins releases by the intraluminal thrombus, biomarkers related to circulating cells and inflammation, metabolomics, and genetic biomarkers. These are described separately in the following section.

\subsection{Biomarkers Related to Extracellular Matrix Homeostasis or Proteolysis}

3.1.1. Cystatin $C$ and Cathepsin. Cystatin $\mathrm{C}$ is the endogenous inhibitor of the elastolytic enzymes cathepsins, which are strongly expressed in the aneurysmatic wall. Cystatin C expression is found reduced in AAA disease, leading to lack of inhibition of the elastolytic properties of cathepsins. In a prospective study [18], a negative correlation of serum cystatin $\mathrm{C}$ values with AAA size and annual expansion rate was found, but without mentionable potential for predicting cases requiring surgery.

In another large study [19], plasma cathepsin S levels correlated positively with aortic diameters but negatively with lowest ankle-branchial index (ABI). Plasma cathepsin S levels showed no correlation with AAA annual expansion rate, a finding mentioned as inexplicable. After logistic regression analysis, the study also reflected reduced cystatin $\mathrm{C}$ in humans with AAA lesions, while no association was found between cystatin $\mathrm{C}$ and AAA annual growth rate, likely because of the relatively short AAA observation time.

\subsubsection{Circulating Basement-Membrane (BM) Fragments.} Types IV and XVIII of collagen are components of the basement membrane. Ramazani et al. [20] have recently shown that AAA patients had significantly increased levels of types IV and XVIII collagen compared with the controls ( $P=0.005$ and $P<0.001$, resp.). Moreover, AAA patients had significantly increased level of type XVIII collagen $(P<0.01)$ when compared with the peripheral arterial disease (PAD) group [20].

This study was conducted in a small number of patients, indicating that further studies are required to establish the potential role of BM fragments as biomarkers for AAA.

3.1.3. Osteoprotegerin (OPG). It has been suggested that serum osteoprotegerin (OPG) levels are associated with growth of AAAs while in vitro experiments showed that OPG promotes matrix metalloprotease (MMP) release from monocytes and vascular smooth muscle cells [21, 22]. In a recent study [23], the concentration of aortic wall OPG was positively associated with established markers of AAA severity (cathepsins A, B, and S and the activity of MMP-2 and MMP-9), while it appeared to be associated with lymphocytes and plasma cells. These newer data in humans reinforce the older findings of a role for OPG in AAA pathogenesis.

3.1.4. Tenascin-C (TN-C). TN-C is a matricellular (extracellular matrix) protein that is synthesized by various cell types including vascular smooth muscle cells (VSMC) in response to inflammatory cytokines and mechanical stress [24]. TN-C is typically synthesized in pathological conditions like wounds, inflammation, and tumorigenesis [25]. No biomarker is available for indicating the pathological status of VSMC and interstitial cells and TN-C may be useful for this purpose, possibly in combination with other inflammatory markers and ECM degradation products. TN-C has the advantage of being deposited locally in the inflammatory 
lesion (AAA) and it is also released in stable forms into circulation [26].

Further studies are required to elucidate the complete function of TN-C and to evaluate whether serum levels or bioimaging of TN-C is better suited for the assessment of disease activity in human AAA.

\subsection{Biomarkers Related to Cellular Signaling Pathways}

3.2.1. C-Reactive Protein. It is known that C-reactive protein (CRP) is an independent risk factor for arteriosclerosis.

In a cohort study in men with small AAAs [27], CRP levels were found higher in larger AAAs, but no association was found with the aneurysm expansion rate. As a possible explanation, it was suggested that inflammation, as depicted by the levels of inflammatory markers, is more a response to expansion rather than the primary cause.

In another study [28], no correlation was found between levels of circulating CRP and other inflammatory markers and the expansion of small-diameter AAAs, indicating no clinical use of these markers in AAA surveillance.

A recent cohort study [29] investigated the association between plasma CRP levels and AAA diameter and assessed the relationship between the gradient of CRP levels and rates of expansion in asymptomatic AAAs. A statistical association was confirmed between the AAA diameter and high sensitive-CRP (hs-CRP) plasma levels, showing a possible causal association and suggesting hs-CRP plasma level gradient as a marker of disease progression and rate of expansion.

Since these results are conflicting, further, larger studies are required to draw safe conclusions.

3.2.2. Diminished Soluble Tumor Necrosis Factor-Like Weak Inducer of Apoptosis (sTWEAK). sTWEAK is a type II transmembrane glycoprotein of the TNF superfamily that circulates in plasma [30] and is expressed in SMCs and leukocytes in arterial wall [31]. sTWEAK was found reduced in patients with coronary artery disease [32], carotid atherosclerosis [33], or PAD [34]. Martín-Ventura et al. [35] measured sTWEAK plasma levels in patients with AAA and found that sTWEAK concentrations were decreased in small $(\leq 5 \mathrm{~cm}, P=0.03)$ as well as large AAAs $(>5 \mathrm{~cm}, P=0.004)$ compared with healthy subjects. Moreover, sTWEAK concentrations were negatively associated with AAA size $(P=0.008)$ and AAA expansion rate with 5 years of follow-up $(P=0.031)$ [35].

These results show that sTWEAK is strongly associated with the presence of an aneurysm but fails to differentiate among small and large aneurysms [35].

\subsection{Proteins Released by Intraluminal Thrombus (ILT)}

3.3.1. Peroxiredoxin-1 (PRX-1). Martinez-Pinna et al. [36] analyzed proteins released by intraluminal thrombus (ILT) with proteomic approach and found that PRX-1 was more released by the luminal layer compared with the abluminal layer of the ILT. Increased PRX-1 serum levels in AAA patients compared with healthy subjects and a positive correlation among PRX-1 and AAA diameter and expansion rate were also found. The combination of PRX-1 and AAA size seems to be significantly predictive of AAA growth [36], establishing PRX-1 as a promising biomarker.

3.3.2. Neutrophil Gelatinase-Associated Lipocalin (NGAL). NGAL plasma concentrations have been associated with cardiovascular diseases [37]. Polymorphonuclear cells (PMNs) isolated from AAA patients secreted significantly greater amounts of NGAL than PMNs from controls and correlated with retrospective AAA growth [38]. The ILT releases large amounts of NGAL compared to the abluminal thrombus, the aneurysm wall, and the healthy aortic media [38]. Further studies in larger subjects groups are needed to confirm the association between NGAL and AAA presence and growth [38].

It has been suggested that the ILT of AAAs predisposes for enlargement and rupture. The growth of the AAA is dependent on proteolytic degradation of elastin. NGAL can bind to MMP-9 and inhibit its degradation, thereby preserving enzymatic activity [39]. Complexes of NGAL and active MMP-9 were present in thrombus, the interface fluid, and the aneurysm wall. Still, the importance of these observations is unknown and the contribution of the complex NGAL/MMP9 to the AAA growth should be further evaluated [39].

\subsubsection{Insulin-Like Growth Factors and Their Binding Proteins} (IGFs and IGFBPs). Lindholt et al. [40] have evaluated the potential role of IGF-I and IGF-II as biomarkers in 115 patients with AAA, kept under annual surveillance for 10 years. Serum IGF-I correlated positively with AAA size and growth rate $(P=0.016$ and $P=0.004$, resp.), findings that persist after adjustment for potential confounders. Serum IGF-I level predicted cases needing later surgery (95\% confidence interval (CI):0.52-0.73) [40].

IGFBP-1 was localized in the luminal part of AAA thrombus and IGFBP-1 levels were increased in AAA thrombus conditioned media, compared to media layer and healthy media [41]. It seemed to facilitate the potentiation of ADPinduced platelet aggregation triggered by IGF-1, while its concentrations were significantly higher in large AAA patients compared with control subjects (normal aortic size) $(P<$ 0.01). Moreover, IGFBP-1 levels correlated with AAA size $(P<0.001)$, which remained significant after adjusting for risk factors [41].

\subsection{Biomarkers Related to Circulating Cells and Inflammation}

3.4.1. Lymphocytes. Increasing evidence shows that the autoimmune response contributes importantly to the pathogenesis of AAA. More specifically, CD4(+), CD25(+), and FOXP3(+) T regulatory cells (Tregs) were found significantly decreased in AAA patients compared to the control group $(P<0.01)$, indicating impaired immunoregulation [42].

Additionally, the loss of the inhibitory receptor CD31 on peripheral T lymphocytes is found to be associated with the incidence of atherosclerotic complications such as AAA in patients [43]. These findings should be further researched 
in order to establish potential biomarkers of the natural history of AAA, as well as potential therapeutic targets for the inhibition of the creation and progression of aneurysms.

In another study, increased plasma levels of sCD28 and sCD86 $(P=0.0001)$ and decreased plasma levels of sCTLA$4(P=0.0018)$ were found in AAA patients compared with normal individuals. These levels were not related to the patient's age or the size of aneurysm, but there was a significant inverse relationship between the concentrations of sCTLA-4 and sCD80 with matrix metalloproteinase-9 [44].

3.4.2. Monocytes. In a more recent study [45], CD16(+) monocyte subsets were found increased in large abdominal aortic aneurysms and were differentially related to circulating and cell-associated (CD143) biochemical and inflammatory biomarkers. A clinical implication of this study is that, by taking common blood measurements (plasma D-dimer, creatinine, and age to derive eGFR, uric acid, total white blood, and neutrophil counts), one could discriminate AAA patients with different monocyte-dependent inflammatory profiles. This study was hampered by cross-sectional design and the relatively small number of patients, while it was unable to find a clear relation of the size of AAA and the values of circulating monocyte subsets in patients at different stages of expansion of aortic damage.

3.4.3. Progenitor Cells. CD34(+) levels are a known marker of circulating progenitor cells. Van Spyk et al. [46] investigated the role and compared the percentage of CD34(+) cells in AAA disease and peripheral vascular disease (PVD). This small study revealed a lower percentage of CD34(+) cells in AAA patients, compared to PVD patients, concluding that AAA is a less severe vascular disease than PVD. Further study is needed in order to establish $\mathrm{CD} 34(+)$ cells as a biomarker for risk stratification.

3.4.4. Lymphangiogenesis. A study by Scott et al. [47] attempted to research the relationship between inflammation and neovascularization in AAA tissue. The results showed that the aneurysm wall contained high levels of inflammatory infiltrate, while microvascular densities of blood $(P<$ $0.001)$ and lymphatic $(P=0.003)$ vessels were significantly increased in AAA samples compared with controls. Vascularity as expressed by positive staining for CD31, CD105, and D2-40 correlated positively with inflammation, while increased VEGFR-3 and VEGF-A expression was observed within inflammatory areas of AAAs. These results suggest lymphatic vessel involvement in AAA disease, associated with the extent of inflammation [47].

In a recent study [48], infiltration of lymphatic vessel endothelial hyaluronan receptor- (LYVE-) 1, vascular endothelial growth factor- (VEGF-) C, and matrix metalloproteinase- (MMP-) 9-positive macrophages and podoplanin and Prox-1-positive microvessels were identified by immunohistochemistry in the intima/media in the AAA wall, where hypoxia-inducible factors- (HIF-) $1 \alpha$ was expressed. VEGF-C and MMP-9 were not expressed in macrophages infiltrating in the adventitia. With the intraoperative use of indocyanine green lymphography, lymph stasis was revealed in the intima/medial in AAA patients, while fluorescence microscopy confirmed the accumulation of lymph in the intima/media but not in adventitia. These results demonstrate that infiltration of macrophages in intima/media is associated with lymphangiogenesis and angiogenesis in AAA, while there is evidence of inadequate lymph-drainage in the AAA wall.

3.4.5. Catalase. PMNs play a key role in AAA progression. Diminished catalase expression and activity were observed in PMNs from AAA patients compared with controls. Catalase plasma levels were also decreased in large and small AAAs when compared with healthy individuals. This study was also conducted in a very small number of patients [49].

3.4.6. Pathogens. One study [50] showed that $P$. gingivalis, a common pathogen involved in chronic periodontitis, accelerates AAA progression via recruitment and activation of neutrophils, leading to production of NETs which are detectable in the plasma of AAA subjects. These results were confirmed by an experiment in rats. Since repeated subclinical episodes of bacteremia are systematically associated with periodontal diseases, $P$. gingivalis could be therefore a key factor in human AAA progression. Still, more epidemiological and observational studies in humans should be made before organizing therapeutic strategies based on the treatment of periodontal disease to prevent AAA evolution towards rupture.

3.5. Metabolomics. Metabolomics stands for sensitive analytical techniques such as metabolic fingerprinting with multivariate analysis. Metabolomics seems to be a good approach to find biomarkers of AAA [51].

Guanidinosuccinic acid (GSA), which is mainly released from the ILT, is highly increased in the plasma of AAA patients when compared to controls. GSA behaves like nitric oxide (NO), due to its vasodilatory actions and its ability to activate the NO generating $\mathrm{N}$-methyl-D-aspartate (NMDA) receptor. After being generated in the ILT, GSA is secreted to blood stream, and the amount of secreted GSA is related to the stage of AAA [52].

Hippuric acid is secreted only by the luminal part of the ILT and was found significantly decreased in the plasma of AAA patients. This observation correlates with the hyperexcretion of hippuric acid in atherosclerotic state [51].

Long-chain acylcarnitines were decreased in the plasma of AAA patients compared to controls. There was a clear decreasing trend with increasing size of aneurysm which may indicate altered fatty acid $\beta$-oxidation or deficiency of carnitine [51]. Additionally, a significant decrease in sphingosine1-phosphate (S1P) and sphinganine-1-phosphate in AAA patients was also found. Both molecules are sphingolipids; sphinganine-1-phosphate is a parent of sphingosine and S1P. S1P is a lysophospholipid and significant changes in other lysophospholipids like LysoPEs and LysoPCs are reported in this investigation. A possible explanation for decrease in the 
amount of LysoPCs/PEs in plasma of AAA patients, with a trend related to the size of aneurysm, is their accumulation in the ILT [51].

3.5.1. Vitamin D Binding Protein (DBP) and Vitamin D. During last decades, there has been a surge of interest in vitamin $\mathrm{D}$ and its wide range of health benefits, partially due to the many association studies linking vitamin $\mathrm{D}$ status with common human diseases. DBP is the main serum carrier of vitamin $\mathrm{D}$ metabolites, with albumin acting as an alternative lower affinity binder [53]. Gamberi et al. [54] showed a negative correlation between DBP and the presence of AAA. Even if its value is not well established yet, DBP is pivotal for vascular remodeling and it may have an important role in the protection of vascular walls.

In a more recent observational study [55], 4233 older men (70-88 years old) participated in a randomized controlled trial of screening for AAA. The study measured their infrarenal aortic diameter by ultrasound and their $25(\mathrm{OH}) \mathrm{D}$ plasma levels by immunoassay. An inverse relationship between vitamin $\mathrm{D}$ status and the presence of larger AAA was found, along with an inverse dose-response association between $25(\mathrm{OH}) \mathrm{D}$ concentrations and the size of AAA, suggesting a role of vitamin $\mathrm{D}$ in the severity of aneurysmal arterial disease. Further research is needed to clarify the mechanisms underlying these associations.

3.5.2. Homocysteine. Wong et al. [56] investigated in a crosssectional study the relationship of homocysteine and the presence and diameter of AAAs in older men (70-88 years old). Plasma total homocysteine ( $\mathrm{tHcy}$ ) was found to be associated with the presence of AAA, while there was also a positive dose-response relationship between tHcy and abdominal aortic diameter. The investigators concluded that further, longitudinal studies and clinical trials of lowering tHcy are required in order to assess if these relationships are causal.

3.5.3. Lipoproteins and Lipoprotein-Related Receptors. Results of a meta-analysis suggest that circulating lipoprotein alpha $(\mathrm{Lp}(\mathrm{a}))$ concentrations may be higher in patients with AAA than those in subjects without AAA, thus playing a role in the diagnosis of AAA [57]. Low-density lipoprotein receptorrelated protein 1 (LRP1) demonstrated significant association with AAA size $(P=0.0042)$ [58]. In a small pilot study in 12 patients by Chan et al. [59], lipoprotein receptor-related protein 1 (LRP1) expression was found significantly lower in AAA patients than in controls, while no significant correlation was shown between LRP1 expression and the size of AAA $(P>0.05)$. These results suggest that a reduction in LRP1 expression could be associated with aneurysm progression.

Other metabolites used to discriminate the natural history of patients with large aneurysm, small aneurysm, and controls are the metabolites of cholesterol. Decreased plasma levels of those metabolites were observed in patients with large and small aneurysms in comparison to controls [51]. Lower serum HDL cholesterol and higher serum LDL cholesterol may be associated with the presence of
AAA [60]. The serum HDL concentrations were lower in patients with AAAs and were independently associated with a reduced risk of having an AAA, in men not receiving current lipid-modifying therapy (95\% CI $0.56-0.93$ per $0.4-\mathrm{mM}$ increase) and in the total cohort (95\% CI $0.63-$ 0.91 per $0.4-\mathrm{mM}$ increase). The concentrations of $\mathrm{LDL}$ and triglycerides were not associated with the presence of AAAs [61].

Past studies [62] have revealed decreased low-density lipoprotein receptor-related protein 5 (LRP5) gene expression in peripheral blood cells of AAA patients and an association between decreased expression of LRP5 and increased lipoprotein (a) [Lp(a)] levels in AAA patients. In a recent study [63], LRP5 gene polymorphisms rs4988300 and rs3781590 were found independent genetic markers of AAA, even after adjusting for age, sex, dyslipidemia, hypertension, smoking habit, and chronic obstructive pulmonary disease. AAA patients had significantly higher Lp(a) levels than control subjects $(P<0.0001)$. Further studying of the role of these markers in AAA and of LRP5 gene in $\mathrm{Lp}(\mathrm{a})$ catabolism and AAA pathophysiology is necessary.

3.5.4. Phospholipases. Wallinder et al. [64] showed that patients with small AAAs had increased levels of the enzyme glycosylphosphatidylinositol-specific phospholipase D (GPIPLD) compared with controls without aneurysm by using a proteomic approach, providing some evidence of the value of GPI-PLD as a novel potential plasma biomarker for the detection of AAAs [64]. In the same tone, Golledge et al. found [65] that serum secretory phospholipase $A(2)$ (sPLA(2)) activity is elevated in men with small AAAs but is not associated with AAA progression.

3.5.5. Iron. A recent study by Martinez-Pinna et al. [66] found increased red blood cell- (RBC-) borne iron retention and transferrin, transferrin receptor, and ferritin expression in AAA tissue compared to controls. In contrast, decreased circulating iron, transferrin, mean corpuscular haemoglobin concentration (MCHC), and haemoglobin concentration, along with circulating RBC count, were observed in AAA patients compared to controls, whereas hepcidin concentrations were increased in AAA subjects. Moreover, iron, transferrin, and haemoglobin levels were negatively, and hepcidin positively, correlated with aortic diameter in AAA patients. MCHC negatively correlated with thrombus area in another cohort of AAA patients. Anemia was significantly more prevalent in AAA patients compared to that in patients with atherosclerotic aortoiliac occlusive disease. Finally, the mortality risk among AAA patients with anemia was increased by almost 30 as compared to AAA subjects with normal hemoglobin levels. This study showed that local iron retention and altered iron recycling associated with high hepcidin and low transferrin systemic concentrations could lead to reduced circulating haemoglobin levels in AAA patients and that low haemoglobin levels are independently associated with AAA presence and clinical outcome. 


\subsection{Genetic Biomarkers}

3.6.1. Telomere Length. Telomeres are specialized DNA sequences at the ends of linear chromosomes. Telomere attrition is the phenomenon of telomere length "shortening" with each successive cell division, eventually leading to cell senescence and/or apoptosis. These observations can contribute to the estimation of the cellular biological age [67]. In humans, reduced telomere length in circulating leucocytes is associated with premature vascular disease, with the telomere:genomic DNA content being significantly reduced in wall biopsies of AAA compared to normal aortas $(P<0.001)$ [67]. This decreased telomerase endothelial expression implies a protective role of telomerase against AAA formation [68]. Lowest telomere restriction fragment (TRF) length values double the risk of having AAA compared with a mean TRF length in the highest values $(P=0.005)$ [69].

3.6.2. AAA1 Locus on Chromosome 19q13. Several singlenucleotide polymorphisms (SNPs) were nominally associated with AAAs $(P<0.05)$ [70, 71]. The SNPs with most significant $P$ values were peptidase D (PEPD) and CD22 [71]. Immunohistochemical staining for CD22 revealed protein expression in lymphocytes present in the aneurysmal aortic wall only and no detectable expression in control aortas [71]. PEPD protein was expressed in fibroblasts and myofibroblasts in the media-adventitia border in both aneurysmal and nonaneurysmal tissue samples [71].

3.6.3. 9p21. AAA is among a number of vascular disorders to be recently associated with a common allelic variant situated on chromosome 9p21 [72, 73]. A significant association between rs10757278-G and the presence of AAA was found $(P=0.03)$, an effect size completely consistent with that originally reported [73]. rs10757278 was not significantly associated with altered AAA growth rate [73]. In a newer study [74], single-nucleotide polymorphisms rs10757278 and rs1333049 of chromosome 9p21-3 region were significantly associated with increased risk of AAA. This study revealed no association between polymorphisms and aortic diameters in AAA patients, while a specific genotype (GG of rs10757278) was suggested to interact with the homocysteine biological pathway to stimulate the presence of AAA. This data emphasized the need to further study the role of these biomarkers.

3.6.4. Chemokine Receptors (CCRs). Chronic inflammation plays an important role in AAA formation. The chemokine receptors CCR2, CCR5, and CXCR3 are associated with pathways implicated previously in aneurysm pathogenesis. Chemokine receptor-2 (CCR2) is involved in the regulation of the inflammatory response [75]. CCR2 heterozygote V64I polymorphism and allele frequency were more frequently observed in the AAA group $(P=0.01, P=0.004)$ [75], but there was no significant difference with the control group in relation to the Delta32 allele frequency [76].

Table 1 summarizes the published studies on biomarkers in AAA and their clinical value.

\section{Discussion}

In this review article, we aimed to give a description of the emerging biomarkers that can correlate with biological processes associated with the existence (presence/diagnosis) and progression (size/risk of rupture) of abdominal aortic aneurysms (AAAs). Our main goal was to enrich the current knowledge on biomarkers in AAA disease, as it was presented by Urbonavicius et al. [17] in their review at 2008, and analyze the progress made in this scientific field from 2008 to date. As stated earlier, the clinical value of biomarkers is based on their properties to satisfy the goals of early detection (screening), surveillance in terms of early and later progression, and monitoring of the biologic performance of an AAA after surgical treatment. An ideal biomarker should be able to be applied in all of the above. Early detection and close surveillance can greatly benefit patients, since they are referred for elective repair with a lower risk, compared with the emergency setting [2-4]. The mortality of elective AAA repair has been $5 \%$ or less, but, once rupture occurs, operative mortality is as high as $48 \%$ [77]. Studies have also shown increased cost of open repair of ruptured AAAs (rAAAs) compared to elective open repair; some are even as high as five times more per life saved per year, with the costs from emergency repair of rAAA via EVAR to be even higher $[78,79]$. Many developed countries like the USA, UK, and Sweden have established national screening programs for AAA based mostly on ultrasonography with important results in the field of early detection and follow-up [11-14, 80]. Unfortunately, this is not the case in other countries, which cannot afford such high cost of implementing such a wide screening program [80]. More specifically, in a recent study [81], it was shown that the individual cost per invited subject was $€ 60$ (US \$83.2) and 0.011 additional quality adjusted life years (QALY) were gained per patient in the screened cohort, corresponding to an incremental cost-effectiveness ratio (ICER) of $€ 5673 /$ QALY (US \$7870/QALY). These numbers are unlikely to be feasible in a period of world economic crisis. Taking into account the relatively high prevalence of the disease and the high mortality rates in cases of rupture, along with the high ICU and hospital costs [82], even after EVAR, it seems imperative to establish and standardize biomarkers that can be applied in daily practice and be cost-effective.

As presented, numerous biomarkers, related to the AAA disease, are currently being researched. Most of these studies are either experimental or hampered by their low numbers of patients. Still, one can say that biomarkers reflect biological processes associated with a disease, making them more interesting for personalized medicine rather than for a statistical point of view.

sTWEAK is strongly correlated with aneurysm existence or expansion rate but fails to differentiate small to large aneurysms [35]. TN-C contributes in stratifying risk in patients with AAA before intervention or after EVAR [2], but further study is required to elucidate the function of TN-C and to evaluate whether serum levels or bioimaging of TN$\mathrm{C}$ would be suited for the assessment of disease activity in human AAAs. 


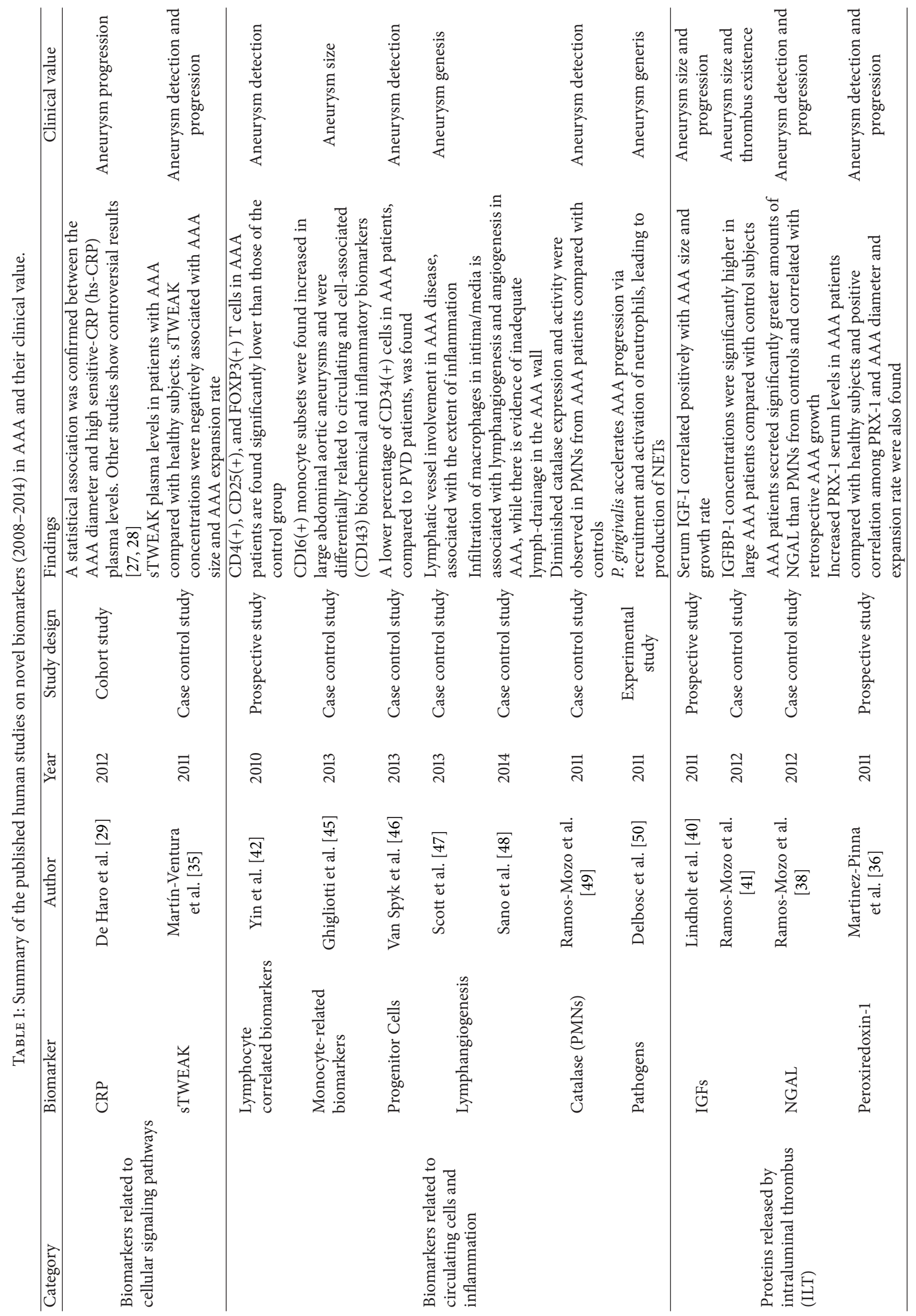




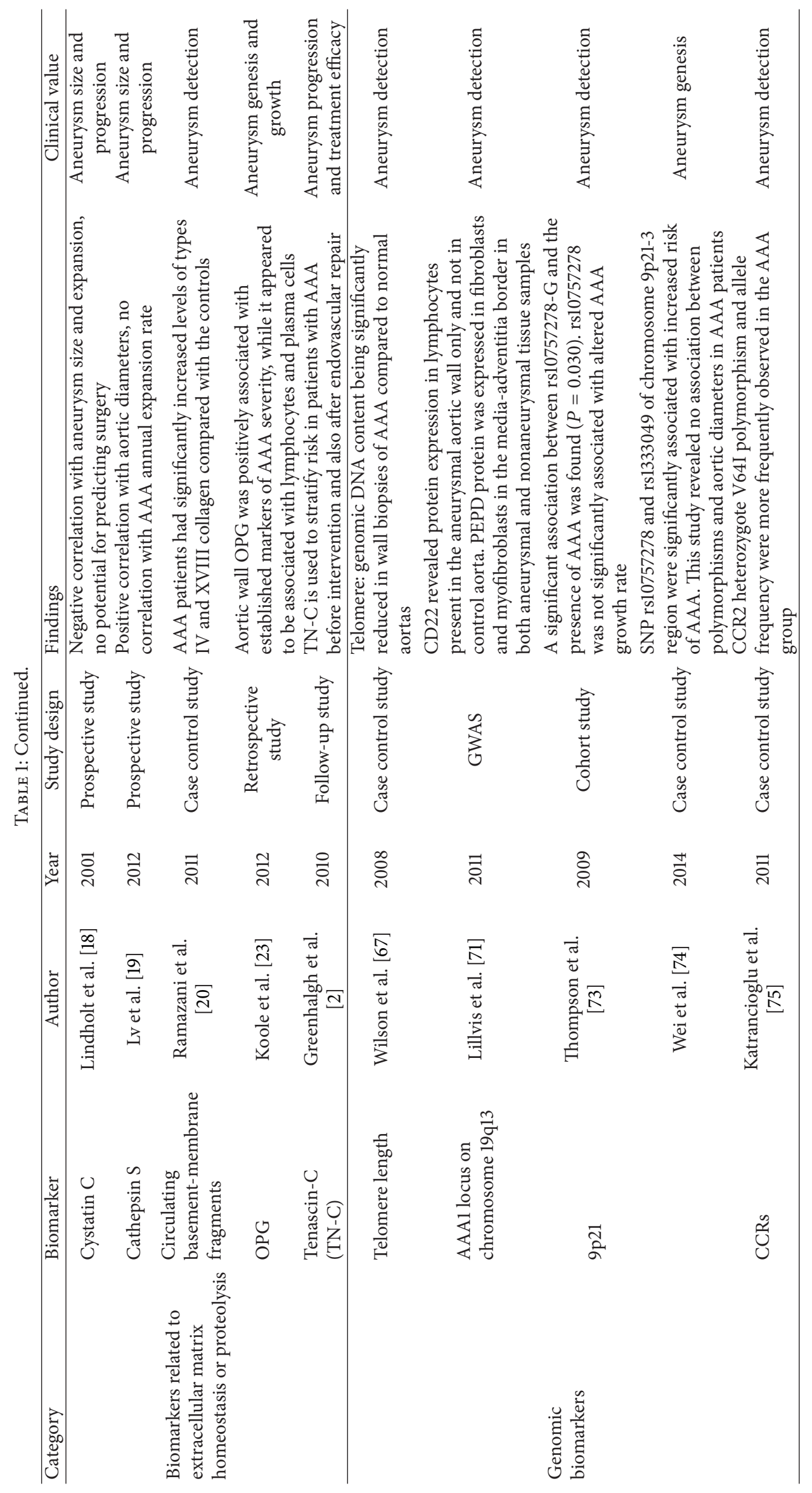




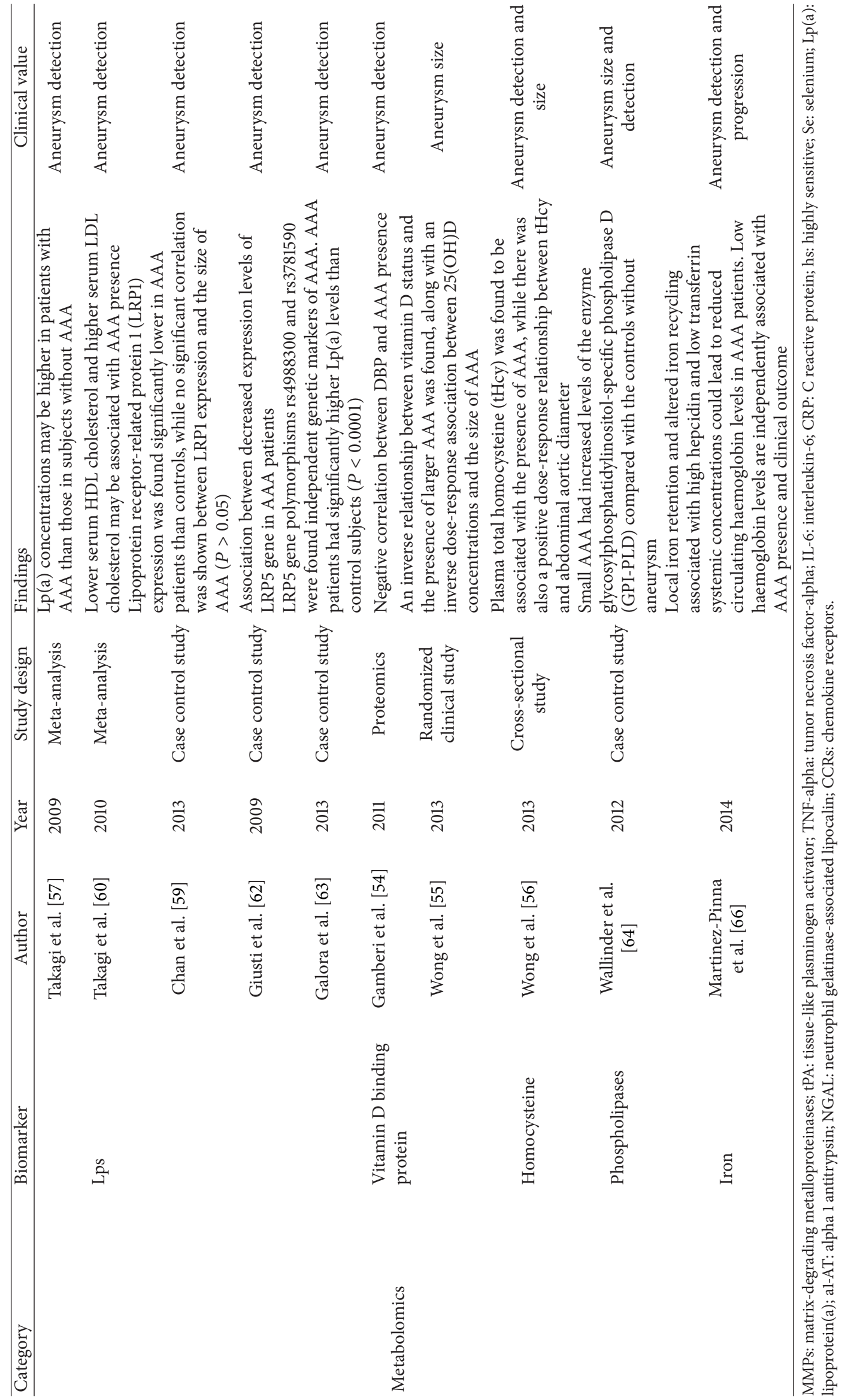


As far as biomarkers related to ILT are concerned, IGF and PRX-1 seem to be the most promising due to their statistically established correlation with AAA diameter and growth $[36,40]$.

Genetic factors did not offer statistically significant results, even though genetic biomarkers remain a wide and undiscovered research field especially as far as the application of chromosome 12 loci in AAA, not just in cases of aortic dissection [83].

As far as metabolomics is concerned, they are probably beneficial in terms of early detection of AAA, but it is not clear in literature how they are affected and in turn biased from the metabolic status of AAA patients (many of them are smokers or suffer from hypertension or/and dyslipidemia, which are risk factors for developing AAA). Therefore, identified metabolites could be good targets for the early detection of AAA [51].

However, none of the aforementioned biomarkers can adequately present the combination of all the pathophysiological events that generate and expand an AAA. This means that there is no biomarker simultaneously indicative of ILT presence, inflammation, and proteolysis.

\section{Conclusions}

Biomarkers may help to explain pathological processes of AAA existence and expansion and allow us to find novel therapeutic strategies or to determine the efficiency of current therapies. To date, there are no specific laboratory markers, which allow us to screen for the disease and monitor its progression or the results of treatment. Further studies and studies in larger patient groups are required in order to validate biomarkers as cost-effective tools in the AAA disease. Advances in modern science guide medicine towards minimal or noninvasive techniques for the diagnosis and management of diseases. Surgery and in particular abdominal aortic aneurysms are no exception and less invasive techniques, like EVAR, are already gaining ground, compared to older methods. This technological progress will hopefully make biomarkers a reality for screening, monitoring, and choosing the optimal time of intervention in abdominal aneurysms.

\section{Conflict of Interests}

The authors declare that there is no conflict of interests regarding the publication of this paper.

\section{Authors' Contribution}

Demetrios Moris and Eleftherios Mantonakis equally contributed and were responsible for the search of the literature and the composition of the paper. Efthymios Avgerinos was responsible for editing the paper and contributing to its composition. Marinos Makris was responsible for drafting the paper and contributing to its composition. Chris Bakoyiannis was responsible for editing and reviewing the paper. Emmanuel Pikoulis was responsible for editing and reviewing the paper. Sotirios Georgopoulos was responsible for the critical and thoughtful review and the supervision of the composition of the paper.

\section{References}

[1] J. Cornuz, C. S. Pinto, H. Tevaearai, and M. Egger, "Risk factors for asymptomatic Abdominal Aortic Aneurysm: sytematic review and meta-analysis of population-based screening studies," European Journal of Public Health, vol. 14, no. 4, pp. 343-349, 2004.

[2] R. M. Greenhalgh, D. J. Allison, P. R. F. Bell et al., "Endovascular versus open repair of Abdominal Aortic Aneurysm," The New England Journal of Medicine, vol. 362, no. 20, pp. 1863-1871, 2010.

[3] D. A. Harris, A. Al-Allak, J. Thomas, and A. R. Hedges, "Influence of presentation on outcome in Abdominal Aortic Aneurysm repair," European Journal of Vascular and Endovascular Surgery, vol. 32, no. 2, pp. 140-145, 2006.

[4] A. D. Dueck, D. S. Kucey, K. W. W. Johnston, D. Alter, and A. Laupacis, "Long-term survival and temporal trends in patient and surgeon factors after elective and ruptured Abdominal Aortic Aneurysm surgery," Journal of Vascular Surgery, vol. 39, no. 6, pp. 1261-1267, 2004.

[5] R. Scott, "A comparative study of the prevalence of Abdominal Aortic Aneurysms in the United Kingdom, Denmark, and Australia," Journal of Medical Screening, vol. 8, no. 1, pp. 46-50, 2001.

[6] F. L. Moll, J. T. Powell, G. Fraedrich et al., "Management of Abdominal Aortic Aneurysms clinical practice guidelines of the European society for vascular surgery," European Journal of Vascular and Endovascular Surgery, vol. 41, no. 1, pp. S1-S58, 2011.

[7] R. M. Greenhalgh, "Endovascular aneurysm repair and outcome in patients unfit for open repair of Abdominal Aortic Aneurysm (EVAR trial 2): randomised controlled trial," Lancet, vol. 365, no. 9478, pp. 2187-2192, 2005.

[8] R. M. Greenhalgh, "Endovascular aneurysm repair versus open repair in patients with abdominal aortic aneurym (EVAR trial 1): randomised controlled trial," Lancet, vol. 365, no. 9478, pp. 2179-2186, 2005.

[9] J. D. Blankensteijn, S. E. C. A. De Jong, M. Prinssen et al., "Two-year outcomes after conventional or endovascular repair of Abdominal Aortic Aneurysms," The New England Journal of Medicine, vol. 352, no. 23, pp. 2398-2405, 2005.

[10] E. L. Chaikof, D. C. Brewster, R. L. Dalman et al., "The care of patients with an Abdominal Aortic Aneurysm: the Society for Vascular Surgery practice guidelines," Journal of Vascular Surgery, vol. 50, no. 4, pp. S2-S49, 2009.

[11] T. C. Hartshorne, C. N. McCollum, J. J. Earnshaw, J. Morris, and A. Nasim, "Ultrasound measurement of aortic diameter in a national screening programme," European Journal of Vascular and Endovascular Surgery, vol. 42, no. 2, pp. 195-199, 2011.

[12] S. Svensjö, M. Björck, M. Gürtelschmid, K. Djavani Gidlund, A. Hellberg, and A. Wanhainen, "Low prevalence of Abdominal Aortic Aneurysm among 65-year-old swedish men indicates a change in the epidemiology of the disease," Circulation, vol. 124, no. 10, pp. 1118-1123, 2011.

[13] F. A. Lederle, G. R. Johnson, S. E. Wilson et al., "Yield of repeated screening for Abdominal Aortic Aneurysm after a 4year interval," Archives of Internal Medicine, vol. 160, no. 8, pp. 1117-1121, 2000. 
[14] A. R. Brady, S. G. Thompson, F. G. R. Fowkes, R. M. Greenhalgh, and J. T. Powell, "Abdominal Aortic Aneurysm expansion: risk factors and time intervals for surveillance," Circulation, vol. 110, no. 1, pp. 16-21, 2004.

[15] R. C. Becker, "Emerging paradigms, platforms, and unifying themes in biomarker science," Journal of the American College of Cardiology, vol. 50, no. 18, pp. 1777-1780, 2007.

[16] R. Limet, N. Sakalihassan, and A. Albert, "Determination of the expansion rate and incidence of rupture of Abdominal Aortic Aneurysms," Journal of Vascular Surgery, vol. 14, no. 4, pp. 540$548,1991$.

[17] S. Urbonavicius, G. Urbonaviciene, B. Honoré, E. W. Henneberg, H. Vorum, and J. S. Lindholt, "Potential Circulating Biomarkers for Abdominal Aortic Aneurysm Expansion and Rupture-a Systematic Review," European Journal of Vascular and Endovascular Surgery, vol. 36, no. 3, pp. 273-280, 2008.

[18] J. S. Lindholt, E. J. Erlandsen, and E. W. Henneberg, "Cystatin C deficiency is associated with the progression of small Abdominal Aortic Aneurysms," British Journal of Surgery, vol. 88, no. 11, pp. 1472-1475, 2001.

[19] B. J. Lv, J. S. Lindholt, X. Cheng, J. Wang, and G. P. Shi, "Plasma cathepsin S and cystatin C levels and risk of Abdominal Aortic Aneurysm: a randomized population-based study," PLoS One, vol. 7, no. 7, article e41813, 2012.

[20] M. Ramazani, C. Lundin, and M. Sund, "Increased circulating levels of basement-membrane components in patients with Abdominal Aortic Aneurysms-A Pilot Study," European Journal of Vascular and Endovascular Surgery, vol. 42, no. 4, pp. 484487, 2011.

[21] C. S. Moran, M. McCann, M. Karan, P. Norman, N. Ketheesan, and J. Golledge, "Association of osteoprotegerin with human Abdominal Aortic Aneurysm progression," Circulation, vol. 111, no. 23, pp. 3119-3125, 2005.

[22] C. S. Moran, P. Clancy, E. Biros et al., "Association of PPAR $\gamma$ allelic variation, osteoprotegerin and Abdominal Aortic Aneurysm," Clinical Endocrinology, vol. 72, no. 1, pp. 128-132, 2010.

[23] D. Koole, R. Hurks, A. Schoneveld et al., "Osteoprotegerin is associated with aneurysm diameter and proteolysis in Abdominal Aortic Aneurysm disease," Arteriosclerosis, Thrombosis, and Vascular Biology, vol. 32, no. 6, pp. 1497-1504, 2012.

[24] E. J. Mackie, T. Scott-Burden, A. W. A. Hahn et al., "Expression of tenascin by vascular smooth muscle cells: alterations in hypertensive rats and stimulation by angiotensin II," American Journal of Pathology, vol. 141, no. 2, pp. 377-388, 1992.

[25] K. S. Midwood and G. Orend, "The role of tenascin-C in tissue injury and tumorigenesis," Journal of Cell Communication and Signaling, vol. 3, no. 3-4, pp. 287-310, 2009.

[26] T. Kimura, K. Yoshimura, H. Aoki et al., "Tenascin-C is expressed in Abdominal Aortic Aneurysm tissue with an active degradation process," Pathology International, vol. 61, no. 10, pp. 559-564, 2011.

[27] P. Norman, C. A. Spencer, M. M. Lawrence-Brown, and K. Jamrozik, "C-reactive protein levels and the expansion of screendetected Abdominal Aortic Aneurysms in men," Circulation, vol. 110, no. 7, pp. 862-866, 2004.

[28] L. Karlsson, D. Bergqvist, J. Lindbäck, and H. Pärsson, "Expansion of small-diameter Abdominal Aortic Aneurysms is not reflected by the release of inflammatory mediators IL-6, MMP9 and CRP in plasma," European Journal of Vascular and Endovascular Surgery, vol. 37, no. 4, pp. 420-424, 2009.
[29] J. De Haro, F. Acin, S. Bleda, C. Varela, F. J. Medina, and L. Esparza, "Prediction of asymptomatic Abdominal Aortic Aneurysm expansion by means of rate of variation of C-reactive protein plasma levels," Journal of Vascular Surgery, vol. 56, no. 1, pp. 45-52, 2012.

[30] Y. Chicheportiche, P. R. Bourdon, H. Xu et al., "TWEAK, a new secreted ligand in the tumor necrosis factor family that weakly induces apoptosis," The Journal of Biological Chemistry, vol. 272, no. 51, pp. 32401-32410, 1997.

[31] B. Muñoz-García, J. L. Martín-Ventura, E. Martínez et al., "Fn14 is upregulated in cytokine-stimulated vascular smooth muscle cells and is expressed in human carotid atherosclerotic plaques: modulation by atorvastatin," Stroke, vol. 37, no. 8, pp. 20442053, 2006.

[32] E. Chorianopoulos, K. Jarr, H. Steen, E. Giannitsis, N. Frey, and H. A. Katus, "Soluble TWEAK is markedly upregulated in patients with ST-elevation myocardial infarction and related to an adverse short-term outcome," Atherosclerosis, vol. 211, no. 1, pp. 322-326, 2010.

[33] L. M. Blanco-Colio, J. L. Martín-Ventura, B. Muñóz-García et al., "Identification of soluble tumor necrosis factor-like weak inducer of apoptosis (sTWEAK) as a possible biomarker of subclinical atherosclerosis," Arteriosclerosis, Thrombosis, and Vascular Biology, vol. 27, no. 4, pp. 916-922, 2007.

[34] J. A. Moreno, T. Dejouvencel, J. Labreuche et al., "Peripheral artery disease is associated with a high CD163/TWEAK plasma ratio," Arteriosclerosis, Thrombosis, and Vascular Biology, vol. 30, no. 6, pp. 1253-1262, 2010.

[35] J. L. Martín-Ventura, J. S. Lindholt, J. A. Moreno et al., "Soluble TWEAK plasma levels predict expansion of human Abdominal Aortic Aneurysms," Atherosclerosis, vol. 214, no. 2, pp. 486-489, 2011.

[36] R. Martinez-Pinna, P. Ramos-Mozo, J. Madrigal-Matute et al., "Identification of peroxiredoxin-1 as a novel biomarker of Abdominal Aortic Aneurysm," Arteriosclerosis, Thrombosis, and Vascular Biology, vol. 31, no. 4, pp. 935-943, 2011.

[37] A. Prabhu, D. I. Sujatha, B. Ninan, and M. A. Vijayalakshmi, "Neutrophil gelatinase associated lipocalin as a biomarker for acute kidney injury in patients undergoing coronary artery bypass grafting with cardiopulmonary bypass," Annals of Vascular Surgery, vol. 24, no. 4, pp. 525-531, 2010.

[38] P. Ramos-Mozo, J. Madrigal-Matute, M. Vega de Ceniga et al., "Increased plasma levels of NGAL, a marker of neutrophil activation, in patients with Abdominal Aortic Aneurysm," Atherosclerosis, vol. 220, no. 2, pp. 552-556, 2012.

[39] M. Folkesson, M. Kazi, C. Zhu et al., "Presence of NGAL/MMP9 complexes in human Abdominal Aortic Aneurysms," Thrombosis and Haemostasis, vol. 98, no. 2, pp. 427-433, 2007.

[40] J. S. Lindholt, J. L. Martin-Ventura, S. Urbonavicius et al., "Insulin-like growth factor i-a novel biomarker of Abdominal Aortic Aneurysms," European Journal of Vascular and Endovascular Surgery, vol. 42, no. 5, pp. 560-562, 2011.

[41] P. Ramos-Mozo, C. Rodriguez, C. Pastor-Vargas et al., "Plasma profiling by a protein array approach identifies IGFBP-1 as a novel biomarker of Abdominal Aortic Aneurysm," Atherosclerosis, vol. 221, no. 2, pp. 544-550, 2012.

[42] M. Yin, J. Zhang, Y. Wang et al., "Deficient CD4+CD25+ T regulatory cell function in patients with Abdominal Aortic Aneurysms," Arteriosclerosis, Thrombosis, and Vascular Biology, vol. 30, no. 9, pp. 1825-1831, 2010. 
[43] G. Fornasa, M. Clement, E. Groyer et al., "A CD31-derived peptide prevents angiotensin II-induced atherosclerosis progression and aneurysm formation," Cardiovascular Research, vol. 94, no. 1, pp. 30-37, 2012.

[44] P. Sakthivel, V. Shively, M. Kakoulidou, W. Pearce, and A. K. Lefvert, "The soluble forms of CD28, CD86 and CTLA4 constitute possible immunological markers in patients with Abdominal Aortic Aneurysm," Journal of Internal Medicine, vol. 261, no. 4, pp. 399-407, 2007.

[45] G. Ghigliotti, C. Barisione, S. Garibaldi et al., "CD16(+) monocyte subsets are increased in large Abdominal Aortic Aneurysms and are differentially related with circulating and cell-associated biochemical and inflammatory biomarkers," Disease Markers, vol. 34, no. 2, pp. 131-142, 2013.

[46] E. N. Van Spyk, K. C. Chun, K. M. Samadzadeh, J. H. Peters, and E. S. Lee, "Increased levels of CD34+ cells are associated in patients with Abdominal Aortic Aneurysms compared with patients with peripheral vascular disease," The Journal of Surgical Research, vol. 184, no. 1, pp. 638-643, 2013.

[47] D. J. Scott, C. J. Allen, C. A. Honstvet et al., "Lymphangiogenesis in Abdominal Aortic Aneurysm," British Journal of Surgery, vol. 100, no. 7, pp. 895-903, 2013.

[48] M. Sano, T. Sasaki, S. Hirakawa et al., "Lymphangiogenesis and angiogenesis in Abdominal Aortic Aneurysm," PLoS One, vol. 9, no. 3, article e89830, 2014.

[49] P. Ramos-Mozo, J. Madrigal-Matute, R. Martinez-Pinna et al., "Proteomic analysis of polymorphonuclear neutrophils identifies catalase as a novel biomarker of Abdominal Aortic Aneurysm: potential implication of oxidative stress in Abdominal Aortic Aneurysm progression," Arteriosclerosis, Thrombosis, and Vascular Biology, vol. 31, no. 12, pp. 3011-3019, 2011.

[50] S. Delbosc, J.-M. Alsac, C. Journe et al., "Porphyromonas gingivalis participates in pathogenesis of human Abdominal Aortic Aneurysm by neutrophil activation. proof of concept in rats," PLoS ONE, vol. 6, no. 4, article e18679, 2011.

[51] M. Ciborowski, J. Teul, J. L. Martin-Ventura, J. Egido, and C. Barbas, "Metabolomics with LC-QTOF-MS permits the prediction of disease stage in aortic abdominal aneurysm based on plasma metabolic fingerprint," PLoS ONE, vol. 7, no. 2, article e31982, 2012.

[52] K. Aoyagi, S. Shahrzad, S. Iida et al., "Role of nitric oxide in the synthesis of guanidinosuccinic acid, an activator of the $\mathrm{N}$-methyl-D-aspartate receptor," Kidney International, Supplement, vol. 59, no. 78, pp. S93-S96, 2001.

[53] D. D. Bikle, E. Gee, and B. Halloran, "Assessment of the free fraction of 25-hydroxyvitamin $\mathrm{D}$ in serum and its regulation by albumin and the vitamin D-binding protein," Journal of Clinical Endocrinology and Metabolism, vol. 63, no. 4, pp. 954-959, 1986.

[54] T. Gamberi, M. Puglia, F. Guidi et al., "A proteomic approach to identify plasma proteins in patients with Abdominal Aortic Aneurysm," Molecular BioSystems, vol. 7, no. 10, pp. 2855-2862, 2011.

[55] Y. Y. Wong, L. Flicker, B. B. Yeap, K. A. Mccaul, G. J. Hankey, and P. E. Norman, "IS hypovitaminosis D associated with Abdominal Aortic Aneurysm, and is there a dose-response relationship?" European Journal of Vascular and Endovascular Surgery, vol. 45, no. 6, pp. 657-664, 2013.

[56] Y. Y. Wong, J. Golledge, L. Flicker et al., "Plasma total homocysteine is associated with Abdominal Aortic Aneurysm and aortic diameter in older men," Journal of Vascular Surgery, vol. 58, no. 2, pp. 364-370, 2013.
[57] H. Takagi, H. Manabe, N. Kawai, S.-N. Goto, and T. Umemoto, "Circulating lipoprotein(a) concentrations and Abdominal Aortic Aneurysm presence," Interactive Cardiovascular and Thoracic Surgery, vol. 9, no. 3, pp. 467-470, 2009.

[58] M. J. Bown, G. T. Jones, S. C. Harrison et al., "Abdominal Aortic Aneurysm is associated with a variant in low-density lipoprotein receptor-related protein 1," The American Journal of Human Genetics, vol. 89, no. 5, pp. 619-627, 2011.

[59] C. Y. Chan, Y. C. Chan, B. L. Cheuk, and S. W. Cheng, "A pilot study on low-density lipoprotein receptor-related protein-1 in Chinese patients with Abdominal Aortic Aneurysm," European Journal of Vascular and Endovascular Surgery, vol. 46, no. 5, pp. 549-556, 2013.

[60] H. Takagi, H. Manabe, N. Kawai, S.-N. Goto, and T. Umemoto, "Serum high-density and low-density lipoprotein cholesterol is associated with Abdominal Aortic Aneurysm presence: a systematic review and meta-analysis," International Angiology, vol. 29, no. 4, pp. 371-375, 2010.

[61] J. Golledge, F. van Bockxmeer, K. Jamrozik, M. McCann, and P. E. Norman, "Association between serum lipoproteins and Abdominal Aortic Aneurysm," American Journal of Cardiology, vol. 105, no. 10, pp. 1480-1484, 2010.

[62] B. Giusti, L. Rossi, I. Lapini et al., "Gene expression profiling of peripheral blood in patients with Abdominal Aortic Aneurysm," European Journal of Vascular and Endovascular Surgery, vol. 38, no. 1, pp. 104-112, 2009.

[63] S. Galora, C. Saracini, A. M. Palombella et al., "Low-density lipoprotein receptor-related protein 5 gene polymorphisms and genetic susceptibility to Abdominal Aortic Aneurysm," Journal of Vascular Surgery, vol. 58, no. 4, pp. 1062-1068, 2013.

[64] J. Wallinder, J. Bergström, and A. E. Henriksson, "Discovery of a novel circulating biomarker in patients with Abdominal Aortic Aneurysm: a Pilot Study using a proteomic approach," Clinical and Translational Science, vol. 5, no. 1, pp. 56-59, 2012.

[65] J. Golledge, Z. Mallat, A. Tedgui, and P. E. Norman, "Serum secreted phospholipase A2 is associated with Abdominal Aortic Aneurysm presence but not progression," Atherosclerosis, vol. 216, no. 2, pp. 458-460, 2011.

[66] R. Martinez-Pinna, J. S. Lindholt, J. Madrigal-Matute et al., "From tissue iron retention to low systemic haemoglobin levels, new pathophysiological biomarkers of human Abdominal Aortic Aneurysm," Journal of Thrombosis and Haemostasis, vol. 112 , no. 1,2014

[67] W. R. W. Wilson, K. E. Herbert, Y. Mistry et al., "Blood leucocyte telomere DNA content predicts vascular telomere DNA content in humans with and without vascular disease," European Heart Journal, vol. 29, no. 21, pp. 2689-2694, 2008.

[68] D. Dimitroulis, A. Katsargyris, C. Klonaris et al., "Telomerase expression on aortic wall endothelial cells is attenuated in Abdominal Aortic Aneurysms compared to healthy nonaneurysmal aortas," Journal of Vascular Surgery, vol. 54, no. 6, pp. 1778-1783, 2011.

[69] G. Atturu, S. Brouilette, N. J. Samani, N. J. M. London, R. D. Sayers, and M. J. Bown, "Short leukocyte telomere length is associated with Abdominal Aortic Aneurysm (AAA)," European Journal of Vascular and Endovascular Surgery, vol. 39, no. 5, pp. 559-564, 2010.

[70] A. F. Baas, J. Medic, R. Van’t Slot et al., "Association study of single nucleotide polymorphisms on chromosome 19q13 with Abdominal Aortic Aneurysm," Angiology, vol. 61, no. 3, pp. 243247, 2010. 
[71] J. H. Lillvis, Y. Kyo, G. Tromp et al., "Analysis of positional candidate genes in the AAA1 susceptibility locus for Abdominal Aortic Aneurysms on chromosome 19," BMC Medical Genetics, vol. 12, article 14, 2011.

[72] A. Helgadottir, G. Thorleifsson, K. P. Magnusson et al., "The same sequence variant on 9p21 associates with myocardial infarction, Abdominal Aortic Aneurysm and intracranial aneurysm," Nature Genetics, vol. 40, no. 2, pp. 217-224, 2008.

[73] A. R. Thompson, J. Golledge, J. A. Cooper, H. Hafez, P. E. Norman, and S. E. Humphries, "Sequence variant on 9p21 is associated with the presence of Abdominal Aortic Aneurysm disease but does not have an impact on aneurysmal expansion," European Journal of Human Genetics, vol. 17, no. 3, pp. 391-394, 2009.

[74] Y. Wei, J. Xiong, S. Zuo et al., "Association of polymorphisms on chromosome $9 \mathrm{p} 21.3$ region with increased susceptibility of Abdominal Aortic Aneurysm in a Chinese Han population," Journal of Vascular Surgery, vol. 59, no. 4, pp. 879-885, 2014.

[75] N. Katrancioglu, S. Manduz, O. Karahan et al., "The role of the CCR2 gene polymorphism in Abdominal Aortic Aneurysms," Angiology, vol. 62, no. 2, pp. 140-143, 2011.

[76] B. Sandford, M. Bown, N. London, and R. Sayers, "The role of the CCR5 $\triangle 32$ polymorphism in Abdominal Aortic Aneurysms," International Journal of Immunogenetics, vol. 36, no. 4, pp. 199-205, 2009.

[77] M. J. Bown, A. J. Sutton, P. R. F. Bell, and R. D. Sayers, "A metaanalysis of 50 years of ruptured Abdominal Aortic Aneurysm repair," British Journal of Surgery, vol. 89, no. 6, pp. 714-730, 2002.

[78] A. M. Cota, A. A. Omer, A. S. Jaipersad, and N. V. Wilson, "Elective versus ruptured Abdominal Aortic Aneurysm repair: a 1-year cost-effectiveness analysis," Annals of Vascular Surgery, vol. 19, no. 6, pp. 858-861, 2005.

[79] R. J. Hinchliffe, S. W. Yusuf, J. A. Macierewicz, S. T. R. MacSweeney, P. W. Wenham, and B. R. Hopkinson, "Endovascular repair of ruptured Abdominal Aortic Aneurysm-a challenge to open repair? Results of a single centre experience in 20 patients," European Journal of Vascular and Endovascular Surgery, vol. 22, no. 6, pp. 528-534, 2001.

[80] N. Nair, D. Sarfati, and C. Shaw, "Population screening for Abdominal Aortic Aneurysm: evaluating the evidence against screening criteria," New Zealand Medical Journal, vol. 125, no. 1350, pp. 72-83, 2012.

[81] S. Giardina, B. Pane, G. Spinella et al., "An economic evaluation of an Abdominal Aortic Aneurysm screening program in Italy," Journal of Vascular Surgery, vol. 54, no. 4, pp. 938-946, 2011.

[82] U. Sadat, J. R. Boyle, S. R. Walsh, T. Tang, K. Varty, and P. D. Hayes, "Endovascular vs open repair of acute Abdominal Aortic Aneurysms-A systematic review and meta-analysis," Journal of Vascular Surgery, vol. 48, no. 1, pp. 227-236, 2008.

[83] J.-H. Pan, J. S. Lindholt, G. K. Sukhova et al., "Macrophage migration inhibitory factor is associated with aneurysmal expansion," Journal of Vascular Surgery, vol. 37, no. 3, pp. 628635, 2003. 


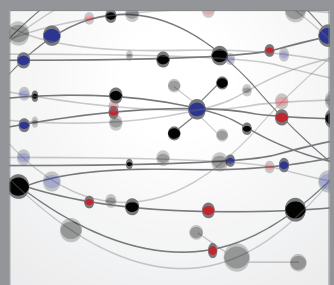

The Scientific World Journal
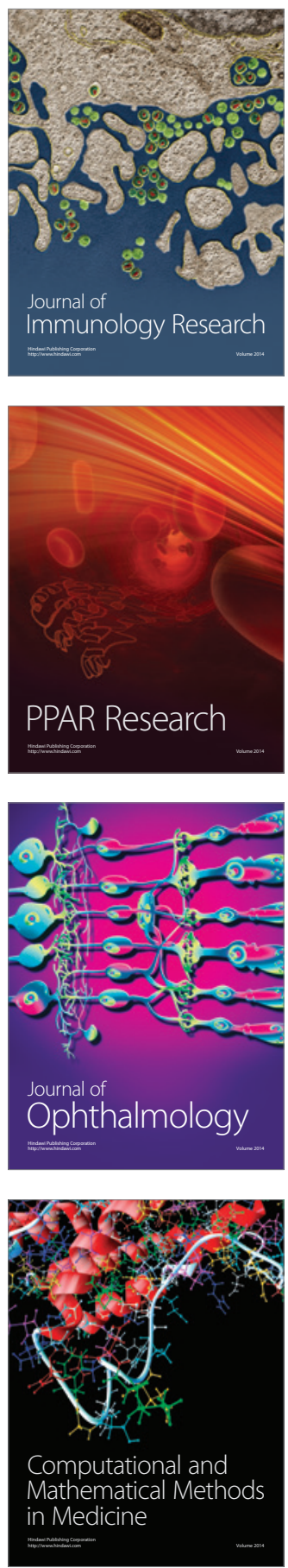

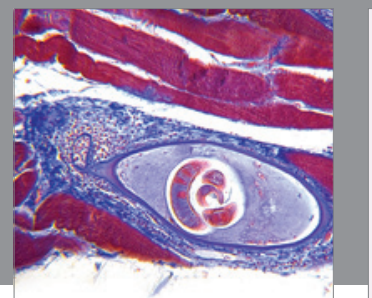

Gastroenterology

Research and Practice
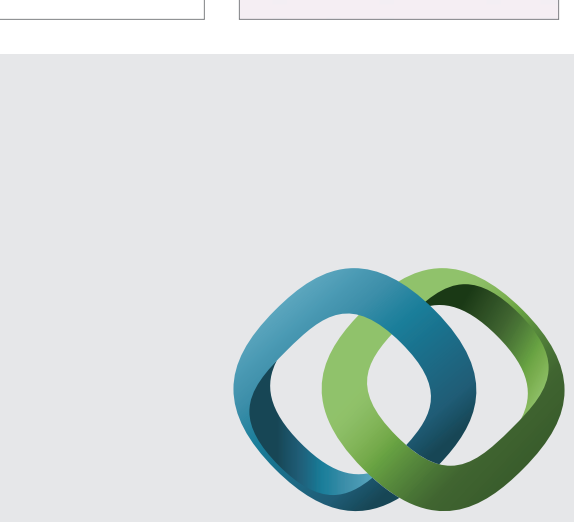

\section{Hindawi}

Submit your manuscripts at

http://www.hindawi.com
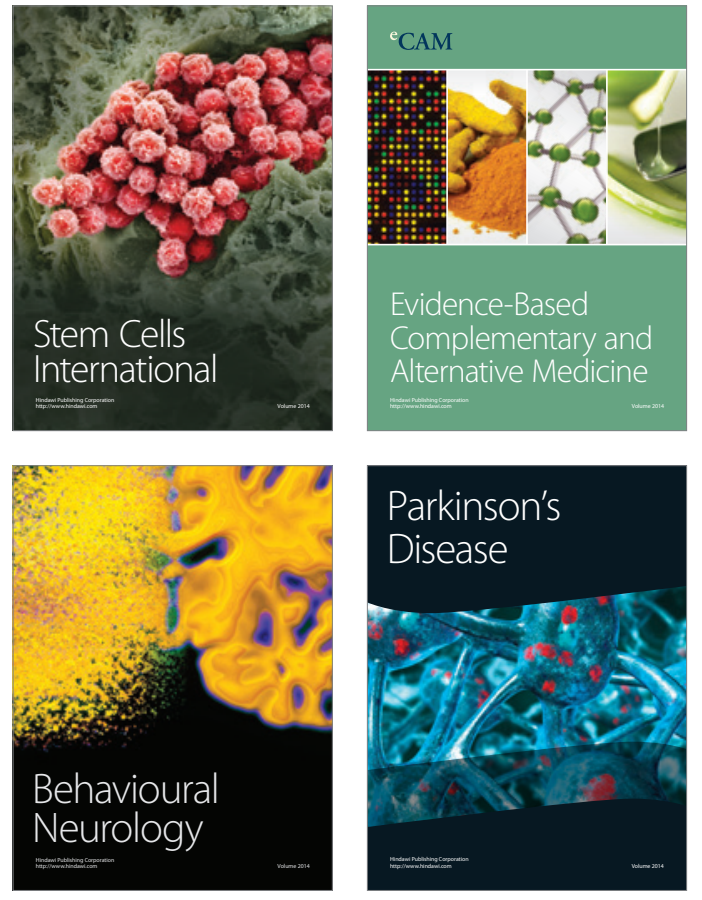
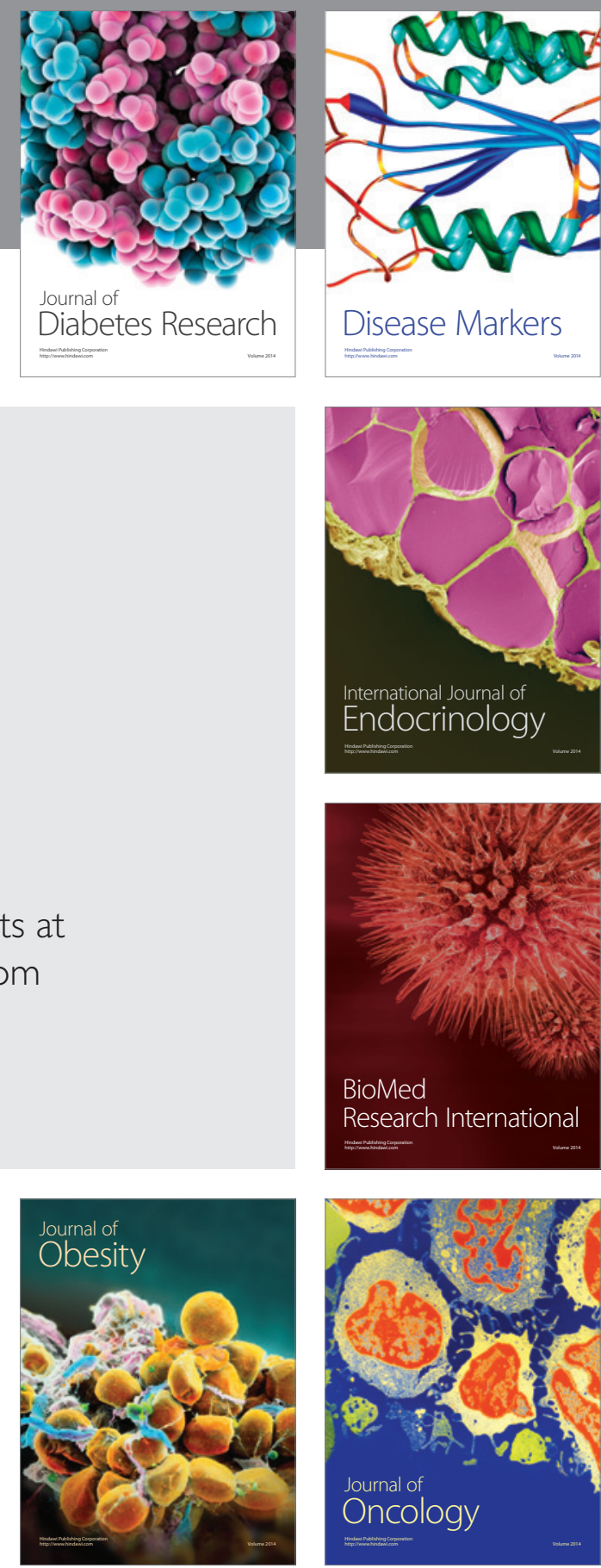

Disease Markers
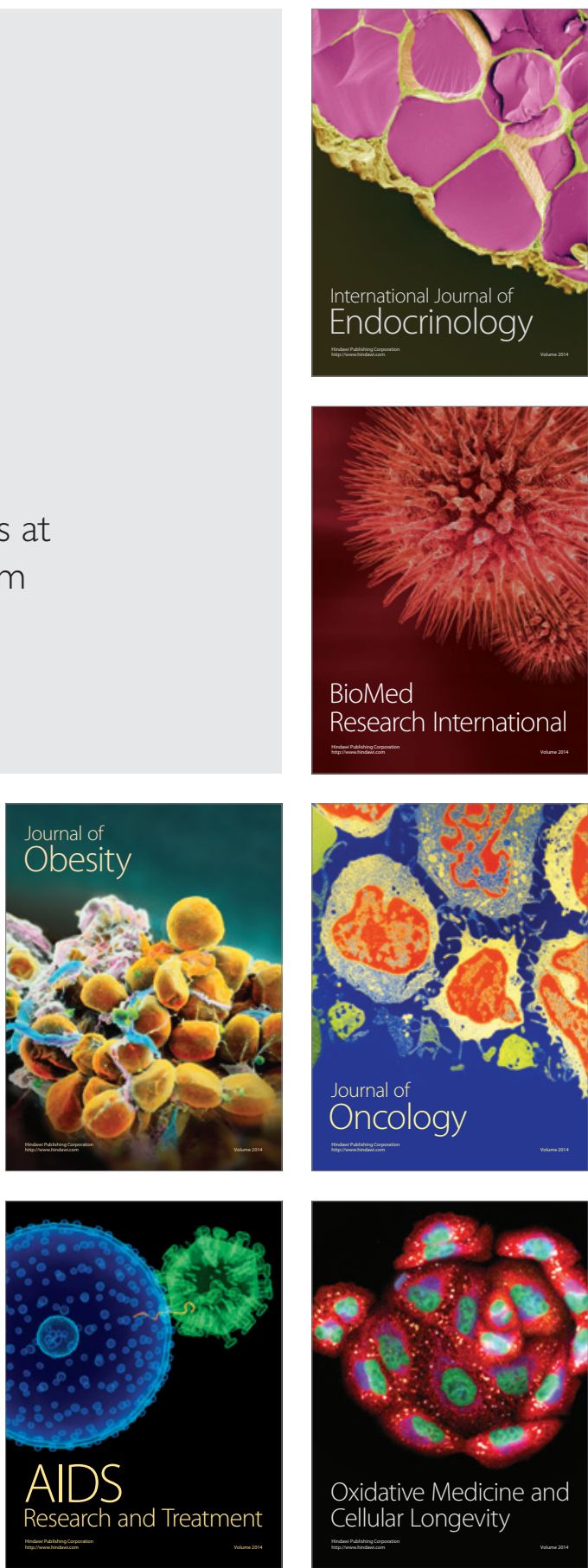